\title{
Российское многоцентровое
}

СКрИнИнГОвое ИссАеАОванИе ПО выявАенИю

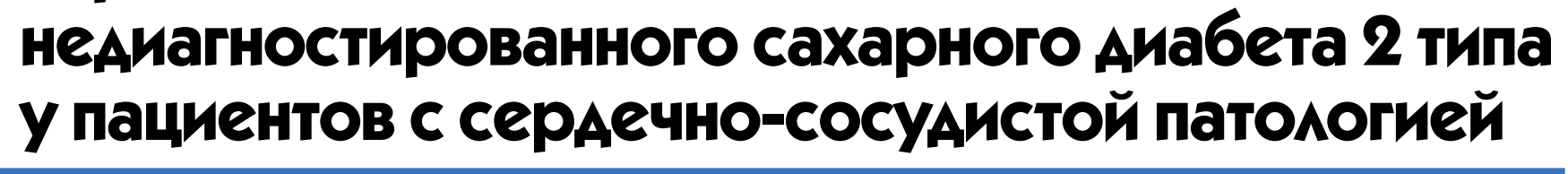

Шестакова М.В. ${ }^{1,2}$, Чазова И.Е. ${ }^{3}$, Шестакова Е.А. ${ }^{1}$ от имени рабочей группы исследования

${ }^{1}$ ФГБУ Эндокринологический научный иентр, Москва ${ }^{2}$ ГБОУ ВПО Первый государственный медицинский университет им. И.М. Сеченова, Москва ${ }^{3}$ ФББУ Российский кардиологический научно-производственный комплекс, Москва

Цель. Оценить распространенность недиагностированного сахарного диабета 2 типа (СД2) среди пациентов с сердечнососудистой патологией.

Материалы и методы. В период с 2013 по 2014 г2. было организовано скрининговое исследование по диагностике СД2 у лии с сердечно-сосудистой патологией в нескольких городах России. В исследование включены пациенты (1001) $\geq 40$ лет, не страдающие СД и имеющие артериальную гипертензию и/или доказанное атеросклеротическое поражение одного из сосудистых бассейнов, посетившие амбулаторный прием кардиолога. Диагностика СД проводилась на основании уровня глюкозы венозной плазмы натощак и/или гликированного гемоглобина (Нb $\left.A_{1}\right)$ и/или результатов перорального глюкозотолерантного теста (ПГТТ). Также оценивался уровень артериального давления (АД), анамнез СД и сердечно-сосудистых заболеваний у родственников, биохимические показатели крови (общий холестерин, холестерин ЛПНП, холестерин ЛПВП, триглицериды).

Результаты. Из 1001 наблюдения глюкоза плазмы натощак была оценена в 1000(99,8\%) случаях, уровень НbА $в$ в 623 (62,2\%) случаях, ПГТТ проведен в 286 (28,6\%) случаях. Распространенность СД по уровню глюкозы плазмы натощак составила

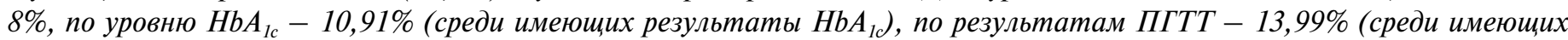
результаты ПГТТ). Предиабетические нарушения углеводного обмена (нарушенная гликемия натощак и нарушенная толерантность к глюкозе) диагностировались в 14,4-36,4\% случаев в зависимости от диагностического метода. Большинство пациентов с СД, выявленных в ходе выполнения ПГТТ, не достигали целевых значений АД и липидного спектра: 67,5\% пациентов имели повышенное систолическое АД, 47,5\% пациентов имели повышенное диастолическое АД, у 90,9\% уровень холестерина ЛПНП превышал 1,8 ммоль/л, у 52,9\% уровень триглицеридов превышал 1,7ммоль/л.

Заключение. Среди пациентов, с сердечно-сосудистой патологией отмечается высокая распространенность недиагностированного СД2 (от $8 \%$ до 13,99\% по данным различных методов диагностики) и предиабетических нарушений углеводного обмена, что может определять необходимость активного скрининга СД у данной категории больных высокого риска.

Ключевые слова: сахарный диабет 2 типа; сердечно-сосудистые заболевания; скрининг

\section{Russian multicentre type 2 diabetes screening program in patients with cardiovascular disease}

Shestakova M.V. ${ }^{1,2}$, Chazova I.E. ${ }^{3}$, Shestakova E.A. ${ }^{1}$, on behalf of the work group

${ }^{1}$ Endocrinology Research Centre, Moscow, Russia

${ }^{2}$ Sechenov First Moscow State Medical University, Moscow, Russia

${ }^{3}$ Russian Cardiological Research and Production Complex, Moscow, Russia

Aim. To evaluate the prevalence of undiagnosed type 2 diabetes mellitus (T2DM) among patients with cardiovascular disease. Materials and methods. T2DM screening programs among patients with cardiovascular disease were held from 2013 to 2014 in several Russian cities. In total, 1001 patients aged $\geq 40$ years with hypertension and/or atherosclerotic disease and without prior diagnosis of T2DM were screened in outpatient cardiology clinics. T2DM diagnosis was based on fasting plasma glucose levels, glycated haemoglobin $\left(\mathrm{Hb}_{1 \mathrm{c}}\right)$ and/or oral glucose tolerance test (OGTT) results. Blood pressure (BP), family history of T2DM, cardiovascular disease, total cholesterol, low-density lipoprotein ( $L D L)$, high-density lipoprotein (HDL) and triglyceride levels were analysed.

Results. Fasting glucose was measured in 1000 (99.8\%) patients, HbA1c was measured in in 623 (62.2\%) and OGTT was performed in 286 (2.6\%). Fasting glucose detected $8 \%$ of newly diagnosed T2DM; among patients who underwent HbA $A_{1 c}$ measurement, the prevalence of T2DM was $10.91 \%$, and among patients who underwent OGTT, the prevalence was $13.99 \%$. Depending on the chosen test, the prevalence of undiagnosed pre-diabetes (impaired fasting glycaemia and impaired glucose tolerance) was in the range of $14.4 \%-36.4 \%$. The majority of patients with T2DM diagnosed by OGTT did not have target blood pressure and lipid levels; $67.5 \%$ had elevated systolic BP, 47.5\% had elevated diastolic BP, 90.9\% had high LDL ( $\geq 1.8 \mathrm{mmol} / \mathrm{l})$ and $52.9 \%$ had high triglyceride levels $(\geq 1.7 \mathrm{mmol} / \mathrm{l})$. 
Conclusion. A high prevalence of undiagnosed T2DM (from $8 \%$ to 13.99\%, depending on the diagnostic criteria) and pre-diabetic state in patients with cardiovascular disease may require screening for T2DM in this high-risk group.

Key words: type 2 diabetes mellitus; cardiovascular disease; screening

DOI: $10.14341 / \mathrm{DM} 7765$

ахарный диабет (СД) и сердечно-сосудистые заболевания (СС3) взаимно отягощают друг друга. Согласно российским и западным рекомендациям, у пациентов с СД общий сердечно-сосудистый риск признается «высоким», а в случае развития поражения органов-мишеней или наличия факторов риска - «очень высоким» $[1,2]$. Более $50 \%$ связанной с СД смертности опосредовано сердечно-сосудистой патологией [3], что требует от врачей эндокринологов и кардиологов совместной работы.

Высокая распространенность недиагностированного СД 2 типа (СД2), относительно большое число пациентов с имеющимися осложнениями на момент постановки диагноза, длительная бессимптомная фаза заболевания являются весомыми аргументами для проведения скрининга. Однако высокая распространенность заболевания не всегда означает эффективность проведения скрининговых программ.

В крупных рандомизированных клинических исследованиях, проведенных с целью оценки эффективности скрининга СД2 как в общей популяции (исследование Ely, 4936 человек), так и у лиц с высоким риском СД (исследование ADDITION-Cambridge, 16047 человек) [4, 5], распространенность недиагностированного СД2 составила от $3 \%$ до 4,5\%. Оба исследования в ходе 10 -летнего наблюдательного периода не продемонстрировали снижения как общей смертности, так и смертности от СС3 или СД у лиц с проведенным скринингом по сравнению с отсутствием скрининга [6].

Тем не менее, скрининг СД может быть оправдан в конкретных популяциях высокого риска, как, например, у женщин с гестационным СД в анамнезе. Как национальные, так и международные клинические рекомендации обозначают необходимость проведения обследования для реклассификации типа СД в послеродовом периоде $[1,7]$.

Пациенты с СС3 также представляют группу высокого риска по развитию СД2. Цель нашего исследования заключалась в оценке распространенности недиагностированного СД2 среди пациентов с кардиологической патологией в рутинной клинической практике.

\section{Цель}

Оценка распространенности недиагностированного СД2 у пациентов с СС3.

\section{Материалы и методы}

Данное исследование являлось скрининговой программой, предполагающей сбор данных для выявления нарушений углеводного обмена у пациентов в Российской Федерации (5 центров в Москве, Рязани, Ижевске, Екатеринбурге, Тюмени).

В исследование включались пациенты $\geq 40$ лет, не страдающие СД, посетившие амбулаторный прием кардиолога и имеющие одно или более из приведенных ниже заболеваний:

- артериальную гипертензию (АГ) I-III степени;

- и/или ИБС (стабильная стенокардия I-IV функционального класса (верифицированная), инфаркт миокарда, чрескожное коронарное вмешательство, операция коронарного шунтирования в анамнезе);

- и/или коронарный атеросклероз (объективные данные (коронароангиография) о поражении коронарных артерий $\geq 50 \%$ );

- $\quad$ и/или острое нарушение мозгового кровообращения в анамнезе;

- и/или верифицированное атеросклеротическое поражение периферических артерий;

- и/или реваскуляризацию артерий нижних конечностей в анамнезе или ампутацию ноги в связи с атеросклеротическим поражением артерий.

Пациенты исключались из исследования при следующих условиях: беременность или кормление грудью; анамнез носительства вируса иммунодефицита человека; лечение от тяжелого аутоиммунного заболевания; хроническое лечение (>30 последовательных дней) пероральными стероидами; тест функции печени, $>3$ раз превышающий норму, на основании недавно измеренного и документально подтвержденного измерения за последние 6 месяцев, если имелись данные. Все включенные пациенты подписали форму информированного согласия.

У включенных пациентов оценивались антропометрические характеристики (рост, вес, индекс массы тела (ИМТ)), семейный анамнез, анамнез СС3 и СД2. При осмотре определялся уровень систолического и диастолического артериального давления (АД). Однократно исследовались биохимические показатели натощак (ряд исследуемых показателей мог быть пропущен): уровень глюкозы венозной плазмы, общего холестерина, холестерина ЛПНП, холестерина ЛПВП, триглицеридов, гликированного гемоглобина $\left(\mathrm{HbA}_{\mathrm{cc}}\right)$.

Диагностика СД проводилась на основании уровня глюкозы венозной плазмы натощак и/или гликированного гемоглобина и/или результатов перорального глюкозотолерантного теста (ПГТТ). ПГТТ проводился в случаях, если: 1) по результатам уровня глюкозы плазмы натощак и гликированного гемоглобина пациент мог быть отнесен к различным группам нарушения углеводного обмена или 2) при нормальных значениях 
Демографическая характеристика участников исследования (M士SD)

\begin{tabular}{|c|c|c|c|}
\hline Показатель & Мужчины, $n=660$ & Женщины, n=341 & Bcero, $n=1001$ \\
\hline \multicolumn{4}{|l|}{ Paca, $\mathrm{n}(\%)$} \\
\hline европеоидная & $651(98,6)$ & $338(99,1)$ & $989(98,8)$ \\
\hline другая & $9(1,4)$ & $3(0,9)$ & $12(1,2)$ \\
\hline Возраст, лет & $61,47 \pm 10,598$ & $67,28 \pm 11,403$ & $63,46 \pm 11,220$ \\
\hline Индекс массы тела, кг/м² & $28,78 \pm 4,867$ & $29,50 \pm 5,063$ & $29,03 \pm 4,944$ \\
\hline Окружность талии, см & $96,70 \pm 13,590$ & $90,38 \pm 13,782$ & $94,55 \pm 13,974$ \\
\hline
\end{tabular}

Таблица 2

Лабораторные показатели липидного профиля (M士SD)

\begin{tabular}{|l|c|c|c|}
\hline Показатель & Мужчины, $\mathrm{n}=660$ & Женщины, $\mathrm{n}=341$ & Bсего, $\mathrm{n}=1001$ \\
\hline Общий холестерин, ммоль/л (n) & $5,12 \pm 1,284(654)$ & $5,54 \pm 1,357(341)$ & $5,27 \pm 1,324(995)$ \\
\hline ХС ЛПНП, ммоль/л (n) & $3,05 \pm 1,062(488)$ & $3,02 \pm 1,038(240)$ & $3,04 \pm 1,053(728)$ \\
\hline ХС ЛПВП, ммоль/л (n) & $1,10 \pm 0,358(494)$ & $1,31 \pm 0,360(248)$ & $1,17 \pm 0,372(742)$ \\
\hline ХС ЛПОНП, ммоль/л (n) & $0,72 \pm 0,457(185)$ & $0,70 \pm 0,640(39)$ & $0,71 \pm 0,492(224)$ \\
\hline Триглицериды, ммоль/л (n) & $1,77 \pm 1,089(563)$ & $1,66 \pm 1,008(263)$ & $1,74 \pm 1,065(826)$ \\
\hline
\end{tabular}

n - количество участников, которым проведено исследование

гликемии натощак врач на основании жалоб пациента мог подозревать СД.

Анализ демографических характеристик участников исследования, кардиоваскулярного диагноза, семейного анамнеза СД и сердечно-сосудистых заболеваний, лабораторных параметров липидного профиля и гликемии, результатов теста толерантности к глюкозе производился в системе статистического анализа и извлечения информации SAS-методами с помощью описательных числовых характеристиких исследуемых переменных (результаты представлены в виде средних и стандартных оклонений, абсолютных и относительных частот).

\section{Результаты}

Всего в программу был включен 1001 пациент. Все пациенты удовлетворяли критериям включения и не имели критериев исключения, а также подписали информированное согласие на участие в исследовании.

Демографическая характеристика участников исследования представлена в табл. 1 .

Среди обследованных больных $66 \%$ составляли мужчины и $34 \%$ женщины, демографические характеристики, а также распространенность СД2 у мужчин и женщин значимо не отличались.

На рис. 1 представлена распространенность курения и ССЗ у включенных в исследование пациентов.

Семейный анамнез СД2 отмечался у 8,5\% мужчин и 13,2\% женщин. СД2 у детей включенных пациентов зарегистрирован не был. Семейный анамнез ССЗ имели $45,5 \%$ мужчин и 44,3\% женщин. СС3 у детей пациентов наблюдались у 5,3\% мужчин и 4,1\% женщин, прошедших скрининг.

Большинство пациентов имели результаты определения липидного профиля (табл. 2). Отсутствие

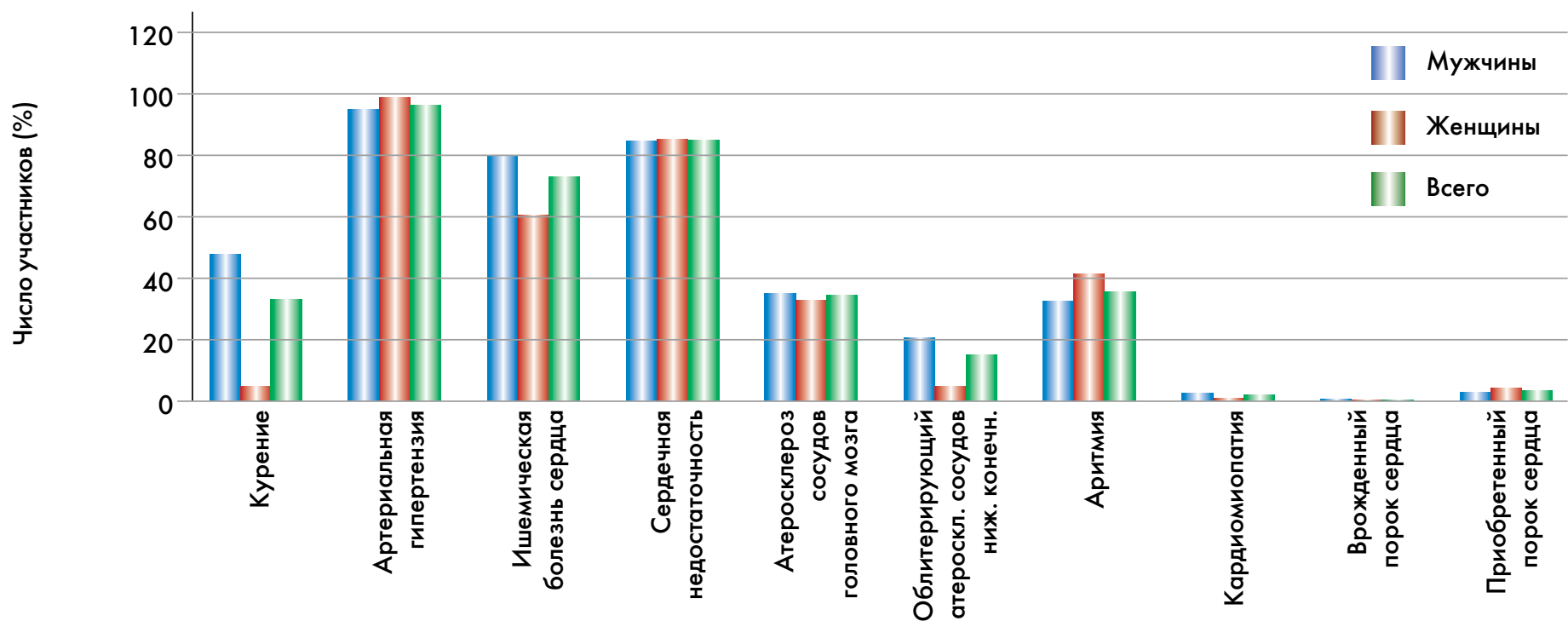

Рис. 1. Распространенность сердечно-сосудистых заболеваний и курения . 
Результаты диагностики нарушений углеводного обмена; n (\%; 95\% ДИ)

\begin{tabular}{|c|c|c|c|c|c|}
\hline \multicolumn{2}{|c|}{ Глюкоза плазмы натощак (n=1000) } & \multicolumn{2}{|c|}{ Гликированный гемоглобин (n=623) } & \multicolumn{2}{|c|}{ Результаты ПГТТ (n=286) } \\
\hline Норма (<6, 1 ммоль/л) & $776(77,6 ; 74,89-80,15)$ & Норма $(<5,7 \%)$ & $328(52,65 ; 48,64-56,63)$ & Норма & $151(52,8 ; 46,83-58,7)$ \\
\hline \multirow{2}{*}{ НГН (6, 1-6,9 ммоль/л) } & \multirow{2}{*}{$144(14,4 ; 12,28-16,73)$} & \multirow{2}{*}{$\begin{array}{c}\text { Группа риска } \\
(5,7-6,4 \%) \\
\end{array}$} & \multirow{2}{*}{$227(36,44 ; 32,65-40,35)$} & $\mathrm{H}$ & 201 \\
\hline & & & & НTГ & 751 \\
\hline ммоль/л) & $80(8 ; 6,39-9,86)$ & СД $(\geq 6,5 \%)$ & $68(10,91 ; 8,58-13,63)$ & СД & $40(13,99 ; 10,18-18,55)$ \\
\hline
\end{tabular}

данных у ряда пациентов допускал дизайн исследования.

Распространенность СД2 среди обследуемых пациентов с имеющимися ССЗ оценивалась по трем критериям: глюкоза венозной плазмы натощак, гликированный гемоглобин, ПГТТ. Из 1001 наблюдения глюкоза плазмы натощак была оценена в 1000 (99,8\%) случаях. Так как исследование проводилось в рутинной клинической практике, то уровень $\mathrm{HbA}_{\mathrm{lc}}$ был измерен только в $623(62,2 \%)$ случаях. У $286(28,6 \%)$ пациентов был проведен ПГТТ. По результатам обследования были выделены следующие группы: без нарушений углеводного обмена (норма), с нарушенной гликемией натощак (НГН), с нарушенной толерантностью к глюкозе (НТГ), с впервые выявленным СД2. Диагностика нарушений углеводного обмена проводилась в соответствии с диагностическими критериями ВОЗ 1999-2013 гг. [1]. Выделение группы риска по уровню гликированного гемоглобина проводилась по критериям Американской диабетической ассоциации 2013 г. [8]. Результаты представлены в табл. 3.

Распространенность СД по уровню глюкозы плазмы натощак составила $8 \%$, по уровню $\mathrm{HbA}_{\mathrm{lc}}-10,91 \%$ (среди имеющих результаты $\mathrm{HbA}_{1 c}$ ), по результатам ПГТТ 13,99\% (среди имеющих результаты ПГТТ). Предиабетические нарушения углеводного обмена (НГН и НТГ) диагностировались в 14,4-36,4\% случаев в зависимости от диагностического метода.

\section{Характеристика пациентов с проведенным ПГТТ}

Среди пациентов с проведенным ПГТТ уровень $\mathrm{HbA}_{1 \mathrm{c}}$ был определен в 80,1\% случаев (у 128 лиц без нарушений углеводного обмена; 9 с НГН, 65 с НТГ и 27 с СД). На рис. 2 приведено распределение уровней $\mathrm{HbA}_{\mathrm{lc}}$ среди

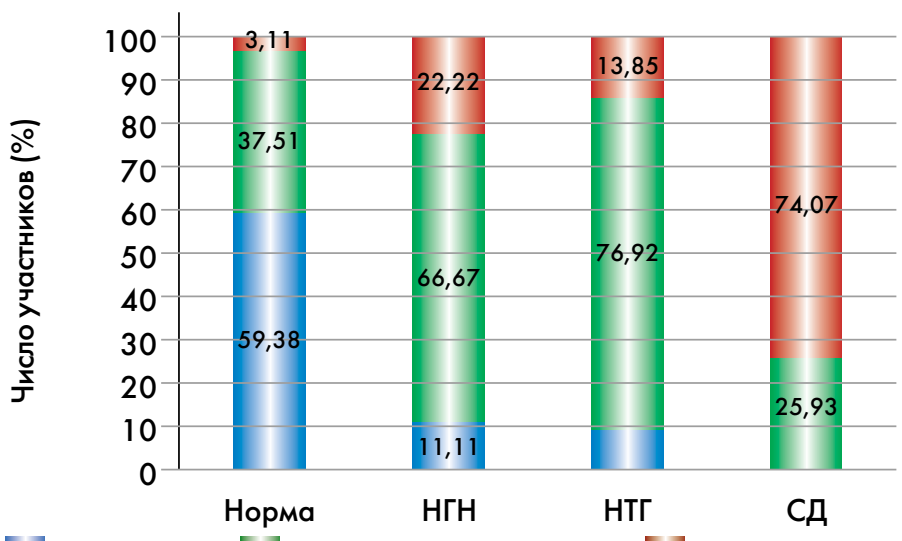

$11 \mathrm{HbA}_{1 \mathrm{c}}<5,7 \| \mathrm{HbA}_{1 \mathrm{c}} \geq 5,7 \% n<6,5 \%$ Рис. 2. Показатель $\mathrm{HbA}_{1 \mathrm{c}}$ среди пациентов с проведенным ПГТТ (B \%). пациентов, имеющих результат ПГТТ. Обращает на себя внимание тот факт, что в группах НГН и СД2 $\mathrm{HbA}_{\mathrm{lc}}$ был измерен в $45 \%$ и $68 \%$ случаев соответственно, что затрудняет интерпретацию результатов.

Наличие избыточной массы тела и ожирения у пациентов с проведенным ПГТТ, определенных на основании подсчета ИМТ, представлена на рис. 3.

Среди пациентов с СД, диагностированным по результатам ПГТТ, высокий уровень ЛПНП ( $\geq 1,8$ ммоль/л) наблюдался в 90,9\% случаев. Сопоставимое количество пациентов с уровнем ЛПНП 1,8 ммоль/л было в группах НГН и НТГ (86,7\% и 90,2\% соответственно). Уровень триглицеридов был измерен у $826(82,5 \%)$ пациентов, причем у 315 из них $(38,1 \%)$ он оказался $\geq 1,7$ ммоль/л

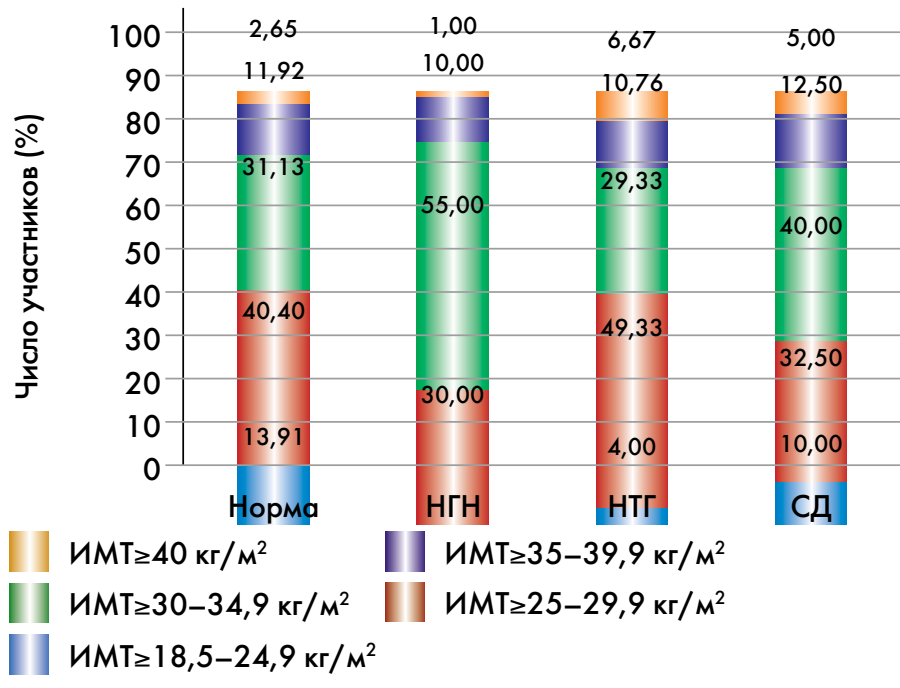

Рис. 3. Распространенность избыточной массы тела и ожирения у пациентов с проведенным ПГТТ.

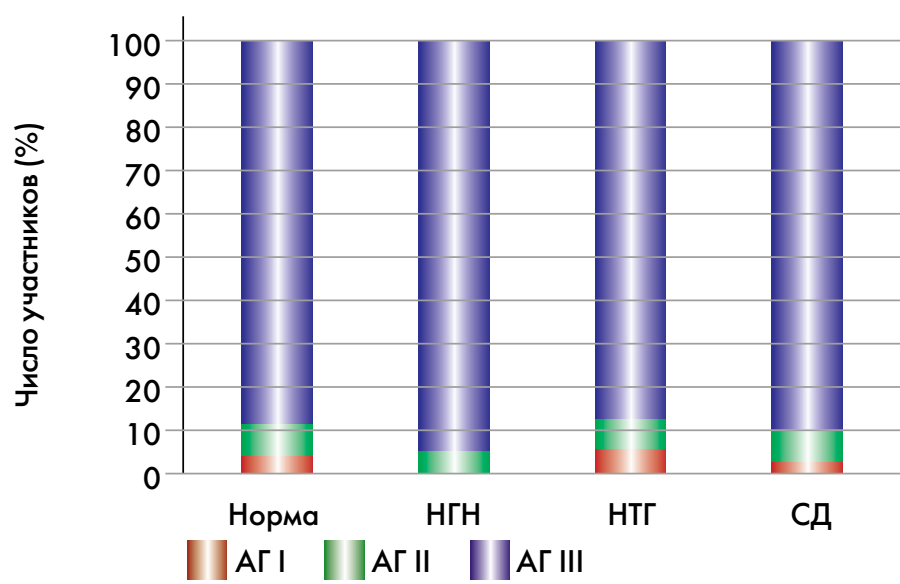

Рис. 4. Распределение артериальной гипертензии по степеням среди лиц с проведенным ПГТТ. 
Сахарный диабет. 2016;19(1):24-29

(у 52,9\% больных СД2, у 55,6\% больных с НГН и 50\% больных с НТГ).

Практически у всех пациентов, у которых имелись результаты ПГТТ, был подтвержден диагноз артериальной гипертензии вне зависимости от типа нарушения углеводного обмена (рис. 4). Следует отметить, что изолированной систолической артериальной гипертензии не было выявлено ни в одной из групп, и в основном преобладала артериальная гипертензия III степени.

\section{Обсуждение}

СД2 представляет собой опасное заболевание с тяжелыми сердечно-сосудистыми последствиями. В связи с длительным бессимптомным его течением и прогнозом интенсивного роста заболеваемости, вопрос о необходимости проведения скрининга СД2 поднимался неоднократно. В исследовании ELSA (English Longitudinal Study of Ageing), проведенном в 2004-2005 гг. в Великобритании среди пациентов с СД 52-79 лет (возрастная категория, схожая с данным исследованием), общая распространенность СД2 составила 9,1\%, среди них 1,7\% впервые диагностированных в ходе исследования [9]. В этом исследовании факторами, ассоциировавшимися с наибольшим риском развития СД, были мужской пол, высокий ИМТ, увеличение окружности талии, уровень АД и триглицеридов. В нашем исследовании, фокусировавшемся на пациентах высокого риска развития СД2 (а именно с СС3 в анамнезе), распространенность впервые выявленного СД, по данным различных методов, составила от $8 \%$ до $13,99 \%$. Следует учесть, что распространенность СД2 по России в общей популяции в возрастной группе от 20 до 79 лет, по данным крупного Российского эпидемиологического исследования NATION, направленного на активный скрининг СД, составляет 5,44\% [10]. Таким образом, распространенность недиагностированного СД2 среди лиц с СС3 выше, чем в общей российской популяции.

Эффективность скрининга в отношении снижения смертности и инвалидизации от СД в нашем исследовании не оценивалась. В рандомизированных исследованиях попытки скрининга СД2 в общей популяции к снижению смертности не привели $[4,5]$. Однако необходимо учесть, что распространенность недиагностированного СД2 в исследовании ADDITION-Cambridge составляла 3\% [4], а в исследовании Ely - 4,5\% [11]. Можно предположить, что в популяции лиц с более высокой распространенностью невыявленного СД, к которым относятся пациенты с СС3, эффективность скрининга в отношении снижения смертности может быть выше.

К отрицательным сторонам скрининга можно отнести психологический вред от появления нового диагноза. Taк, в исследовании ADDITION-Cambridge 116 участников в опросниках указывали на появление психологического дискомфорта в связи с диагностикой у них СД2. В то же время исследования, продолжавшиеся более
1 года, не обнаруживали негативного психологического эффекта проведения скрининга или постановки диагноза СД2 [12, 13].

В нашем исследовании получены различные результаты распространенности впервые выявленного СД по результатам различных диагностических методик. Следует отметить, что прямое сопоставление заболеваемости СД по критериям глюкозы плазмы натощак, уровню гликированного гемоглобина и результатам ПГТТ в данном исследовании не может быть корректным в связи с наличием результатов определения гликированного гемоглобина у $62,3 \%$ участников и результатов ПГТТ только у $28,6 \%$ участников.

Большинство пациентов с СД, по данным ПГТТ, не достигали целевых значений АД и липидного спектра: $67,5 \%$ пациентов имели повышенное систолическое АД, 47,5\% пациентов имели повышенное диастолическое АД, у 90,9\% уровень холестерина ЛПНП превышал 1,8 ммоль/л, у 52,9\% уровень триглицеридов превышал 1,7 ммоль/л.

Данная скрининговая программа имеет ряд ограничений: не была проанализирована зависимость распространенности впервые выявленного СД2 от длительности анамнеза СС3, а также состава и длительности применения антигипертензивной, гиполипидемической, антитромбоцитарной и иной терапии.

\section{Заключение}

Исследование продемонстрировало высокую распространенность недиагностированного СД2 среди пациентов с сердечно-сосудистой патологией, что может определять необходимость активного скрининга СД у данной категории больных.

\section{Дополнительная информация}

Рабочая группа исследования: Калашников В.Ю. (Москва), Якушин С.С. (Рязань), Ополонский Д.В. (Ижевск), Архипов М.В. (Екатеринбург), Шалаев С.В. (Тюмень), Галстян Г.Р. (Москва), Майоров А.Ю. (Москва), Жернакова Ю.В. (Москва), Мельникова О.Г. (Москва)

\section{Информация о финансировании и конфликте интересов}

Исследование проведено при финансовой поддержке компаний «АстраЗенека Фармасьютикалз» и «БристолМайерс Сквибб».

Авторы декларируют отсутствие иных явных и потенциальных конфликтов интересов, связанных с проведением исследования и публикацией настоящей статьи.

Вклад авторов: Шестакова М.В., Чазова И.Е. - координация проекта, редактирование и финальное утверждение рукописи; Шестакова Е.А. - анализ результатов исследования, написание текста. 


\section{Список литературы}

1. Дедов И.И., Шестакова М.В. Галстян Г.Р., и др. Алгоритмь специализированной медицинской помощи больным сахарным диабетом. Под редакцией И.И. Дедова, М.В. Шестаковой (7-й выпуск) // Сахарный диабет. - 2015. - T. 18. - №1S - C. 1-112. [Dedov II, Shestakova MV, Aleksandrov $A A$, et al. Standards of specialized diabetes care. Edited by Dedov II, Shestakova MV (7th edition). Diabetes mellitus. 2018;18(1S):1112. (In Russ)] doi: 10.14341/DM20151S

2. $2013 \mathrm{ESH} / \mathrm{ESC}$ Guidelines for the management of arterial hypertension. European Heart Journal. 2013;34(28):2159-2219. doi: 10.1093/eurhearti/eht151

3. Дедов И.И., Шестакова М.В., Викулова О.К. Государственный регистр сахарного диабета в Российской Федерации: статус 2014 г. и перспективы развития // Сахарный диабет. - 2015. - Т. 18. - №3 C. 5-22. [Dedov II, Shestakova MV, Vikulova OK. National register of diabetes mellitus in Russian Federation. Diabetes mellitus. 2015;18(3):5-22. (In Russ)] doi: 10.14341/DM201535-22

4. Simmons RK, Echouffo-Tcheugui JB, Sharp SJ, et al. Screening for type 2 diabetes and population mortality over 10 years (ADDITION-Cambridge): a cluster-randomised controlled trial. The Lancet. 2012;380(9855):1741-1748. doi: 10.1016/s0140-6736(12)61422-6

5. Simmons RK, Rahman $M$, Jakes RW, et al. Effect of population screening for type 2 diabetes on mortality: long-term follow-up of the Ely cohort. Diabetologia. 2010;54(2):312-319. doi: $10.1007 / \mathrm{s} 00125-010-1949-8$
6. Waugh NR, Shyangdan D, Taylor-Phillips $S$, et al. Screening for type 2 diabetes: a short report for the National Screening Committee. Health Technol Assess. 2013;17(35). doi: 10.3310/hta 17350

7. Diabetes in Pregnancy: Management of Diabetes and lts Complications from Preconception to the Postnatal Period. London 2008.

8. American Diabetes Association. Standards of Medical Care in Diabetes-2013. Diabetes Care. 2013;36(Suppl 1):S1 1-S66. doi: 10.2337/dc13-S011

9. Pierce MB, Zaninotto P, Steel N, Mindell J. Undiagnosed diabetes-data from the English longitudinal study of ageing. Diabet Med. 2009;26(7):679-685. doi: 10.1111/i.1464-5491.2009.02755.x

10. Dedov II, Shestakova MV, Galstyan GR, et al. Study design and methodology of an epidemiological survey to assess the prevalence of type 2 diabetes in Russia: NATION. IDF 2015. Poster 619.

11. Williams DRR, Wareham NJ, Brown DC, et al. Undiagnosed Glucose Intolerance in the Community: the Isle of Ely Diabetes Project. Diabet Med. 1995;12(1):30-35. doi: 10.1111/i.1464-5491.1995.tb02058.x

12. Rahman M, Simmons RK, Hennings SH, et al. Effect of screening for Type 2 diabetes on population-level self-rated health outcomes and measures of cardiovascular risk: 13-year follow-up of the Ely cohort. Diabet Med. 2012;29(7):886-892. doi: 10.1111/i.1464-5491.2012.03570.x

13. Paddison CAM, Eborall HC, French DP, et al. Predictors of anxiety and depression among people attending diabetes screening: A prospective cohort study embedded in the ADDITION (Cambridge) randomized control trial. $\mathrm{Br}$ J Health Psychol. 201 1; 16(1):213-226. doi: 10.1348/135910710×495366
Шестакова Марина В^аАимировна

Шестакова Екатерина Аиексеевна
А.М.Н., проФ., чА.-Корр. РАН, Аиректор ИНСтитута Аиабета ФГБУ «ЭнАОкринологическиЙ научный центр" МинзАрава России, завеАующая КафеАрой энАокринологии и Аиабетологии пеАиатрического факультета ГБОУ ВПО Первый МГМУ им. И.М. Сеченова, Москва, Российская ФеАерация

А.М.Н., проф., чА.-корр. РАН, Аиректор Института КАинической карАиологии

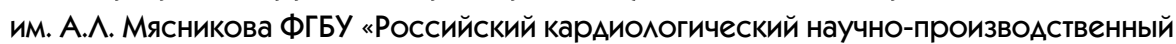
комплекс» Минзарава России, Москва, Российская ФеАераця К.М.Н., научныЙ СотрУАник ОТАеления интервенционной КарАиологии ФГБУ «Энаокринологический научный центр» Минзарава России, Москва, Российская ФеАераця E-mail: katiashestakova@mail.ru

Рукопись получена: 15.12.2015.

Принята к публикации: 18.01.2016. 